\title{
(C) OPEN ACCESS \\ Using routine data to improve palliative and end of life care
}

\author{
Joanna M Davies, ${ }^{1}$ Wei Gao, ${ }^{1}$ Katherine E Sleeman, ${ }^{1}$ Katie Lindsey, ${ }^{2}$ \\ Fliss E Murtagh, ${ }^{1}$ Joan M Teno, ${ }^{3}$ Luc Deliens, ${ }^{4}$ Bee Wee, ${ }^{5}$ \\ Irene J Higginson, ${ }^{1}$ Julia Verne ${ }^{6}$
}

\begin{abstract}
${ }^{1}$ Kings College London, Cicely Saunders Institute, Department Palliative Care, Policy and Rehabilitation, London, UK ${ }^{2}$ National End of Life Care Intelligence Network, Public Health England, London, UK ${ }^{3}$ Division of Gerontology and Geriatric Medicine, Department of Medicine, Cambia Palliative Care Centre of Excellence, University of Washington, Seattle, Washington, USA ${ }^{4}$ End of Life Care Research Group, University of Brussels and Ghent University, Brussels, Belgium

${ }^{5}$ Department of Palliative Care, Experimental Medicine Division, University of Oxford, Oxford, UK ${ }^{6}$ Knowledge \& Intelligence Team (South West), Public Health England, Bristol, UK
\end{abstract}

\section{Correspondence to} Dr Wei Gao, King's College London, Cicely Saunders Institute, Palliative Care, Policy and Rehabilitation, Bessemer Road, London SE5 9PJ, UK; wei.gao@kcl.ac.uk

Received 31 July 2015 Revised 16 October 2015 Accepted 16 December 2015 Published Online First 28 February 2016

\section{CrossMark}

\footnotetext{
To cite: Davies JM, Gao W, Sleeman $\mathrm{KE}$, et al. BMJ Supportive \& Palliative Care 2016:6:257-262.
}

\section{ABSTRACT}

Palliative and end of life care is essential to healthcare systems worldwide, yet a minute proportion of research funding is spent on palliative and end of life care research.

Routinely collected health and social care data provide an efficient and useful opportunity for evaluating and improving care for patients and families. There are excellent examples of routine data research in palliative and end of life care, but routine data resources are widely underutilised. We held four workshops on using routinely collected health and social care data in palliative and end of life care.

Researchers presented studies from the UK, USA and Europe. The aim was to highlight valuable examples of work with routine data including work with death registries, hospital activity records, primary care data and specialist palliative care registers. This article disseminates that work, describes the benefits of routine data research and identifies major challenges for the future use of routine data, including; access to data, improving data linkage, and the need for more palliative and end of life care specific data.

\section{BACKGROUND}

The palliative care resolution adopted by the WHO in 2014 recognises palliative and end of life care (PEoLC) services as essential and integral to health systems worldwide. ${ }^{1}$ However, evidence for effective models of care is limited, not least by current low levels of spending on PEoLC research; $0.24 \%$ of cancer research funding is currently spent on PEoLC research. ${ }^{2}$ Routinely collected health data provides a useful opportunity for evaluating and improving care for patients and families. In December 2013 and March 2014 we held a series of workshops to showcase routine data research in PEoLC, highlight examples of best practice, and stimulate discussion about the potential future use of these resources.

The events were hosted by the National End of Life Care Intelligence Network (NEoLCIN), part of Public Health England (PHE), in partnership with the Cicely Saunders Institute (CSI) at King's College London. One hundred and twenty-five delegates attended the workshops, including researchers from the UK and internationally, representatives from data holding bodies, government organisations, charities, practitioners and experts by experience (also known as service users). The aim of this paper is to describe the current use and the benefits of routine data analysis in PEoLC. The main focus of this paper is on English data, though case studies from Europe and the USA which were presented at the workshops are included to highlight the use of routine data analysis in other healthcare systems. Based on the discussions from workshops, three future priorities are identified for the use of routine data in PEoLC.

\section{WHAT IS ROUTINE DATA?}

Routine data are generated by administrative and clinical processes as opposed to data generated solely for the purposes of research. Death registry data, primary and secondary care data, and other routinely collected health data are increasingly used in PEoLC research. The inherent value of routine data is that it already exists and often on a population basis, negating the challenges associated with primary data collection in PEoLC. Each resource has particular strengths and weaknesses in the context of secondary use in PEoLC research.

The level (person, service or area), or unit that data are collected and made available at has consequences for how the data 
can be used and how it is governed (figure 1). The proportion of home deaths in a region (area level data), or number of specialist palliative care (SPC) consultants based at a hospital (service level data), allow comparisons to be made between areas and services. Person level data contain the richest information about patient's characteristics, their diagnosis and treatments. A combination of area, service and person level data are needed to fully understand healthcare. Patient centred outcome measures (PCOMs), are increasingly seen as the gold standard for measuring quality of care, and require person level data collected uniformly over time.

\section{Death registry data}

Death registry data are an important source of routine data for PEoLC and has been successfully used to draw attention to temporal, spatial and patient variation in place of death. ${ }^{34}$ In most countries a legal requirement to register all deaths means that death registries have complete population coverage. Furthermore, the relative simplicity and consistency of the information collected in death registries, and adherence to international WHO standards on recording causes of death, makes death registry data well suited to historical and international comparisons Box 1.

Place of death has been widely used as a proxy for quality of care. However, there are limitations to what we can learn from the fairly shallow set of crosssectional data contained in death registries. There is now a call to broaden the focus, to measure treatments, preferences, and quality of care directly, to do this we must use different data.

\section{Hospital activity data}

Data collected in hospitals are our main source of information on the diagnosis and treatment received by patients in secondary and tertiary care settings. In England the national hospital activity data set, Hospital Episode Statistics (HES), is beginning to be used in PEoLC studies. ${ }^{6}$ Other national resources on secondary and tertiary care include Cancer Registry data, and the Mental Health and Learning Disabilities data.

Routine data, if sufficiently detailed, can lend itself to use with quasi-experimental methods such as propensity score matching, difference-in-differences, predictive risk modelling, causal networks, and instrumental variable analysis. These methods seek to generate robust evidence on the effects of interventions and treatments, and are particularly useful for building evidence on populations who might be difficult to include in clinical trials such as those in the last days of life, the very frail or cognitively impaired. Box 2 uses American hospital data generated for insurance claims with propensity score matching. The propensity score matching method is a retrospective matching exercise that comprehensively matches a control group to a treatment group alike on all measured characteristics other than the receipt of the treatment or exposure.

\section{Primary care data}

In the UK, general practitioners (GPs) working in primary care are often the first point of contact for patients and around $90 \%$ of the UK population is registered with a GP. Plans to make GP data available nationally in England via the National Health Service (NHS) 'care.data' resource are on hold after confidence in the project was undermined by concerns about privacy of data. The Clinical Practice Research Datalink (CPRD) - previously the General Practice Research Database (GPRD) - is currently the largest available collection of primary care data, containing longitudinal medical records on a sample of around $10 \%$ of the UK population. A recent study using the GPRD confirmed the increasing trend of single and multiple opioid prescribing for patients with cancer at the end of life and highlighted underprescription of opioids to older people. ${ }^{8}$

Other European countries face similar challenges generating accessible, national primary care data. The European Sentinel GP Networks Monitoring End of Life Care (EURO SENTIMELC) project addresses this by collecting data from a representative network of GPs from four countries; Belgium, the Netherlands, Italy and Spain, covering $2 \%-4 \%$ of the general population in each country. ${ }^{9}$ Participating GPs register all deaths on a weekly basis, using a standardised form, generating an epidemiological 'surveillance system' used to compare and monitor end of life care across countries and over time.

\section{Locally available data}

Another major gap in the available national data is the lack of data on social care. Here locally collected data have provided valuable insight. The Nuffield Trust, in collaboration with the NEoLCIN, used data on statefunded community social care from seven local authorities (LA) in England, and linked it at individual level to secondary healthcare data. The project investigated the association between health and social care use in the past 12 months of life, showing wide variation between LAs, and indicated an inverse relationship between social care use and hospital use at the end of life. ${ }^{10}$

\section{National surveys and service monitoring data}

The NHS carries out surveys on large nationally representative samples to monitor service users' experience of care. These surveys are not routine data, but are regularly collected and can be used to examine variation between groups and evaluate policy. For example, a recent study using data from the Views of Informal Carers-Evaluation of Service (VOICES) survey, a mortality follow-back survey, demonstrated that relatives of people aged 85 years and over are less 


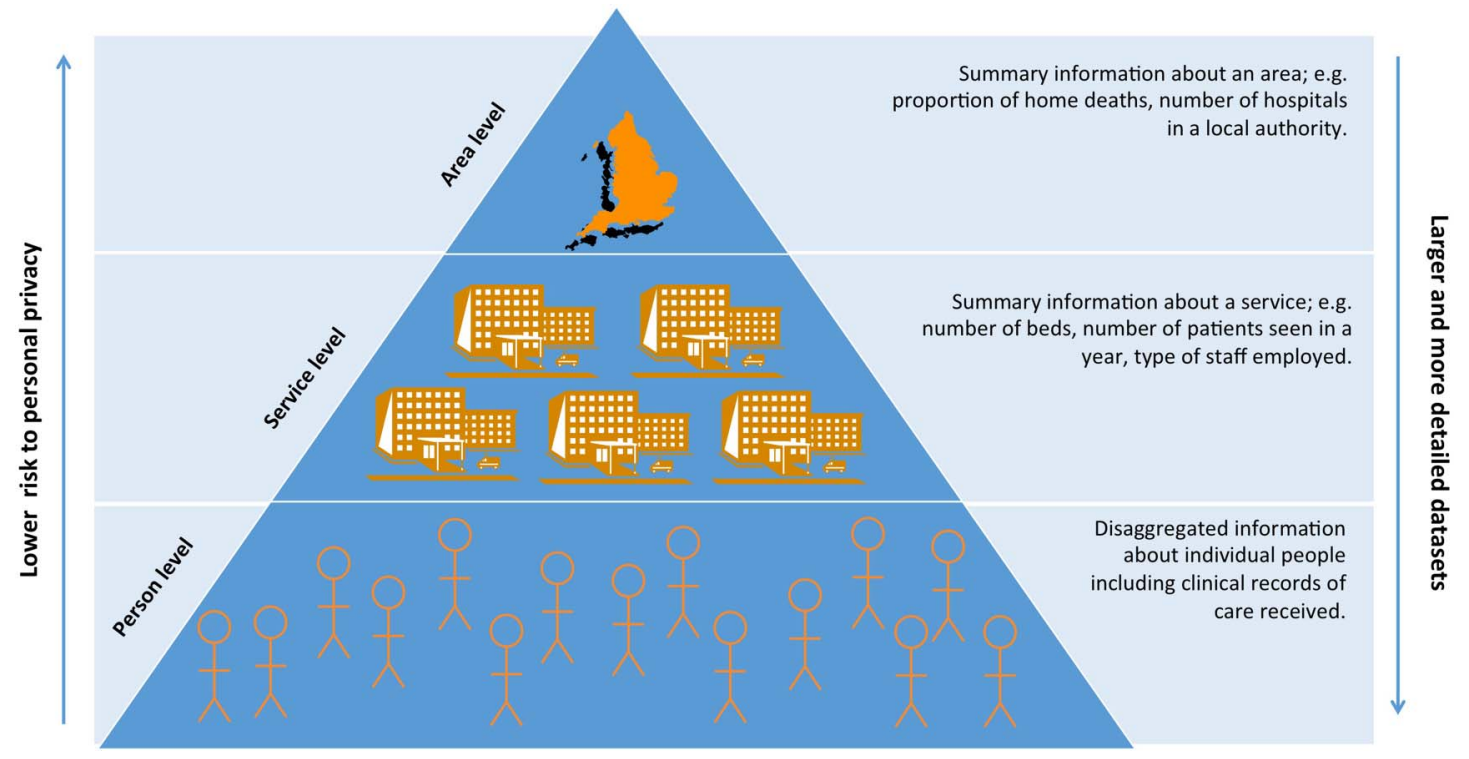

Figure 1 Different units of routinely collected data.

likely than those of younger people to know their loved one's preferences for end of life care, and that this significantly contributes to age-related disparity in place of death. $^{11}$

Much of the monitoring data collected by the NHS, including VOICES and other surveys such as the Carers Survey and the Adult Social Care Survey is publically available, at area level, providing useful sources of open access ecological data (http://www. hscic.gov.uk/searchcatalogue). The NEoLCIN also provides area level data, available to download from their website (http://www.endoflifecare-intelligence. org.uk/end_of_life_care_profiles/), and periodically updates interactive area profiles covering key indicators including place of death. Another useful source of open access aggregate data is the UK Data Service (http://ukdataservice.ac.uk/), a gateway for researchers wanting to access Census derived data, administrative data from social services, education, tax, and social security systems and large health and social survey data. Despite a drive towards more open access, data available in the public domain are usually not provided at individual level.

\section{Methodological challenges}

A challenge for using routine data in research is the varying quality of the data. For example, English death registry data contain no information about ethnicity and poor quality occupation-based social class data, limiting its usefulness for understanding sociodemographic variability in place of death. ${ }^{12}$ Changes in classification and coding practices, such as the introduction in 1993 of 'hospice' as a separate place of death category, and the switch in 2001 to International

\section{Box 1 Place of death of older persons with dementia: a study in five European Countries ${ }^{5}$}

Aim: To compare where people with dementia died across five European countries; Belgium, the Netherlands, England, Wales and Scotland

Methods: Death registry data on all deaths in 2003 of people aged 65 and older were obtained for the five countries, and then linked to area-level data on the number of hospital beds and nursing home beds per 1000 of the population aged over 65

Results: Home death was rare (3-5\%) apart from in Belgium (11\%). Death in hospital was higher in the UK (England 36.0\%; Wales 46.3\%; Scotland $33.9 \%)$ and Belgium (22.7\%). In all countries the majority of dementia patients died in long-term care facilities. The Netherlands had the lowest rate of hospital deaths $(2.8 \%)$ and the highest rate of nursing home deaths $(92.3 \%)$

\begin{tabular}{llcccrrr}
\hline & & Belgium & The Netherlands & England & Wales & Scotland & Total \\
\hline Health resource & Hospital beds/1000 & 5.57 & 3.67 & 3.05 & 3.95 & 4.62 & 3.52 \\
& Nursing home beds/1000 & 4.54 & 10.75 & 8.37 & 4.18 & 7.82 & 8.24 \\
Place of death \% & Home & 11.4 & 3.8 & 3.7 & 3.2 & 5.0 & 4.5 \\
& Hospital & 22.7 & 2.8 & 36.0 & 46.3 & 33.9 & 27.4 \\
& Nursing home & 65.9 & 92.3 & 59.7 & 50.2 & 60.8 & 67.5 \\
& Hospice or other SPC & 0.0 & 0.5 & 0.3 & 0.1 & 0.4 & 0.3
\end{tabular}

Implications of this research: The study suggests that the Dutch model of nursing home provision, characterised by good availability of long-term care facilities with specialist nursing and physician care, could be advantageous for reducing hospital deaths in dementia patients 
Box 2 Does feeding tube insertion and its timing improve survival? ${ }^{7}$

Aim: To test the association between insertion of a feeding tube (FT), and timing of the insertion, with survival in patients with advanced cognitive impairment.

Methods: Data on 36492 nursing home residents with dementia was obtained from the US national mandatory Minimum Data Set for Nursing Homes (MDS-NH), and linked to Medicare health insurance claims data. The data set covered the period 1999-2007.

Propensity score matching was used to match those with a FT (treatment group) to those without a FT (control group) based on sociodemographic variables; evidence of advance care planning including advance directives, do-not-resuscitate orders, do not-hospitalise orders, and any feeding restrictions; pertinent medical diagnoses from the MDS; clinical conditions including dehydration, inability to consume food or fluids, fever, wound infection, weight loss, swallowing problems, chewing problems, syringe feeding, mechanically altered diet, dietary supplementation, the amount of body fat as measured according to body mass index, and presence of a pressure ulcer; measures of functional status and disease severity including activity of daily living score; and two models that predict mortality (Advanced Dementia Prognostic Tool score18 and Changes in Health, End-stage disease and Symptoms and Signs score).

One year survival was compared for the treatment and control groups. For those with a FT, 1 year survival was compared for four cohorts based on the timing of insertion.

Results: 1957 (5.4\%) residents had a FT inserted. No difference in survival was found between those with and those without a FT. For those with a FT, the timing of insertion was also not associated with survival.

Implications of this research: The study provides strong evidence for the ineffectiveness of this highly invasive treatment in this population.

Classifications of Disease, 10th Version (ICD-10), from ICD-9, affects comparability of data over time. Another consideration should be bias in the inclusion or exclusion of particular groups or events from data sets. For example, US Medicare insurance data are largely limited to a population aged over 65 years.

Routine data are prone to problems with inaccurate or inadequate measurement of the issues of interest to researchers, due to the fact that researchers usually have no control over what data are collected or how. This can mean that routine data lack the components to make robust conclusions and adequately control for confounding factors, or chance variability. ${ }^{13}{ }^{14}$ The REpoting of studies Conducted using Observational Routinely collected Data (RECORD) are a useful resource for ensuring quality and transparency in reporting routine data research. ${ }^{15}$

\section{FUTURE PRIORITIES}

Discussion in our workshops identified three major challenges for the use of routine data in PEoLC research: safe and ethical access to data, improving data linkage, and the need for more PEoLC specific data.

\section{Safe and ethical access to data}

There is an on-going debate regarding national health and social data; on the one hand recognition of the power of data to inform better care, and on the other heightened concern about misuse of data and risks to personal privacy. Lack of clarity over the safe and ethical secondary use of data is arguably the biggest barrier preventing better access to routine data for research. In general accessing health and social care data for research purposes in England is a difficult, lengthy and uncertain process. However, there are systems that buck this trend; the Clinical Record Interactive Search (CRIS), based at the National Institute for Health Research (NIHR) Biomedical Research Centre and Dementia Unit (BRC/U), is one example of a system that has been specifically designed to facilitate research access to individual level clinical data, demonstrating that safe and efficient secondary use of data is possible. ${ }^{16}$ The CRIS also uses natural language processing, enabling analysis of free-text patient notes, beyond the scope of traditional data analysis.

\section{Improved data linkage}

Linking data sets is the primary way we can use data more effectively. However, linked data also carries the most risks in terms of potential identifiability and sensitivity of data. In recent years in England there has been a concerted effort via the introduction of trusted third parties (TTP) and the Administrative Data Research Network (ADRN) (previously the Administrative Data Liaison Service (ADLS)) to improve access to linked data (http://adrn.ac.uk/). There are also developments in technology that can aid safe data linking; DataSHIELD is one approach in development that allows remote data pooling. ${ }^{17}$ Another approach is the use of shared pseudonymisation 'keys' to enable multiple data holders to protect the identity of individuals before sharing the data in a linkable form. ${ }^{10}$ The best examples of cross-sectorial data linkage come from Nordic countries. ${ }^{18}$ Key features of the Nordic systems are the widespread use of shared unique identifiers, strong collaboration between data holding bodies, an established legal basis for the collection and use of data, and broad public approval for the use of linked administrative data.

\section{PEoLC specific data}

Population registers collecting data on all patients receiving SPC currently exist in Denmark and Sweden. Plans for a new national, patient level, data 
collection from SPC services in England are currently underway. ${ }^{19}$ The data will include elements from the Integrated Palliative care Outcome Scale (IPOS), a PCOM developed and validated for use with dying patients. This represents a major step forward in our ability to evaluate quality of PEoLC in England.

Two other international SPC data collections are notable; the Australian Palliative Care Outcomes Collaborative (PCOC), ${ }^{20}$ and the Coalition of Hospices Organised to Investigate Effectiveness (CHOICE) in the USA. ${ }^{21}$ An important feature of PCOC and CHOICE is the involvement of healthcare professionals in agreeing the common set of measures to collect and how the data should be used, including strong systems of feeding back the data to services.

We should be mindful that not all patients who could benefit from SPC will receive SPC, and these patients would be excluded from an SPC register. Access to a broad range of data is necessary to understand the PEoLC needs of the whole population.

Quality concerns about existing non-PEoLC specific routine data are pertinent problems. For example the underreporting of specialist palliative care activity in hospital activity data. Where there are quality-related limitations to using existing routine data in PEoLC, surely it is our responsibility to report these fully and identify solutions for improving the data.

\section{NEXT STEPS: PRIORITIES FOR THE BETTER UTILISATION OF ROUTINE DATA IN PEOLC}

The following six points were identified during the workshops as priorities for progressing work with routine data in PEoLC.

Safe and ethical access to data:

1. Strengthen collaborative relationships with data holding bodies; invite representatives to join project advisory groups and attend conferences;

2. Encourage data holding bodies to publish a portfolio of existing projects accessing their data. This could aid transparency, help to avoid repetition and wastage in research, and encourage collaboration across teams and disciplines.

Improved data linkage:

3. Build expertise within PEoLC about safe data linkage techniques that enable more innovative linkage projects;

4. Pursue new and different data sets for linking projects, including locally collected data.

PEoLC specific data:

5. Take responsibility for highlighting limitations of current routine data resources and suggest ways in which these problems might be overcome.

6. Engage with the wider PEoLC community-healthcare professionals, service planners, patients and familieswith the potential benefits routine data can have for improving care. Better engagement can help to improve the quality of existing data collections, and help to inform the development of new resources, and the governance of routine health data.

\section{CONCLUSIONS}

The routine data workshop series benefited from involvement of international colleagues. Nevertheless, a limitation of the work is the predominant focus on English data. An overarching theme has been the benefits we can glean from engagement beyond our immediate colleagues. We hope that the series has been successful in building relationships that will further work with routine data in PEoLC. Above all there is a clear impetus and enthusiasm for increasingly using routine data resources to improve care for patients and families.

Twitter Follow Joanna Davies at @JoannaDavies58

Acknowledgements The authors thank to those who presented or chaired during the workshops: Martin Bardsley, Cynthia Dawn Benz, Sabine Best, Tony Bonser, Rachel Burman, Simon Chapman, Jacquline Crowther, Lorna Fraser, Uy Hoang, Mike Hobday, Melanie Hodson, Dirk Houttekier, John Hughes, Sonali Kaushik, Jonathan Koffman, Paul McCrone, Carolyn Morris, Giovanna Polato, Martin Prince, Malcolm Roxburgh, Keith Sephton, Rob Stewart, Teresa Tate, Katherine Taylor, Keri Thomas. And to people who helped organise and facilitate; Kerry Archer-Dutton, Sian Best, Mendwas Dzingina, Clare Ellis-Smith, Maria Heldakova, Christina Ramsenthaler, Jon Templeton, Fiona Wong. The authors also thank you also to all those who attended.

Contributors JV, IJH, JMD, WG and KES conceived the idea for this paper. JMD and WG wrote the manuscript supported by IJH, JV and KES, with critical revisions by all other authors (KL, FEMM, JT, LD and BW). JMD and WG are jointly responsible for the overall content. This paper does not report original research.

Funding This report is independent research funded by Public Health England, and the National Institute for Health Research Collaboration for Leadership in Applied Health Research \& Care Funding scheme. The views expressed in this publication are those of the author(s) and not necessarily those of the NHS, the National Institute for Health Research or the Department of Health. The Collaboration for Leadership in Applied Health Research and Care (CLAHRC) South London is part of the National Institute for Health Research (NIHR), and is a partnership between King's Health Partners, St. George's, University London, and St George's Healthcare NHS Trust.

Competing interests None declared.

Provenance and peer review Not commissioned; externally peer reviewed.

Open Access This is an Open Access article distributed in accordance with the Creative Commons Attribution Non Commercial (CC BY-NC 4.0) license, which permits others to distribute, remix, adapt, build upon this work noncommercially, and license their derivative works on different terms, provided the original work is properly cited and the use is non-commercial. See: http://creativecommons.org/licenses/by$\mathrm{nc} / 4.0 /$

\section{REFERENCES}

1 WHO. Strengthening of palliative care as a component of integrated treatment within the continuum of care. World Health Organisation. World Health Organisation, 2014.

2 Sleeman KE, Gomes B, Higginson IJ. Research into end-of-life cancer care-investment is needed. Lancet 2012;379:519.

3 Gao W, Ho YK, Verne J, et al. Changing patterns in place of cancer death in England: a population-based study. PLoS Med 2013;10:e1001410. 
4 Sleeman KE, Ho YK, Verne J, et al. Place of death, and its relation with underlying cause of death, in Parkinson's disease, motor neurone disease, and multiple sclerosis: a population-based study. Palliat Med 2013;27:840-6.

5 Houttekier D, Cohen J, Bilsen J, et al. Place of death of older persons with dementia. A study in five European countries. J Am Geriatr Soc 2010;58:751-6.

6 Fraser LK, van Laar M, Miller M, et al. Does referral to specialist paediatric palliative care services reduce hospital admissions in oncology patients at the end of life? Br J Cancer 2013;108:1273-9.

7 Teno JM, Gozalo PL, Mitchell SL, et al. Does feeding tube insertion and its timing improve survival? J Am Geriatr Soc 2012;60:1918-21.

8 Gao W, Gulliford M, Bennett MI, et al. Managing cancer pain at the end of life with multiple strong opioids:

a population-based retrospective cohort study in primary care. PLOS ONE 2014;9:e79266.

9 Ko W, Deliens L, Miccinesi G, et al. Care provided and care setting transitions in the last three months of life of cancer patients: a nationwide monitoring study in four European countries. BMC Cancer 2014;14:960.

10 Bardsley MG, Georghiou T, Dixon J. Social care and hospital use at the end of life. The Nuffield Trust, 2010.

11 Hunt KJ, Shlomo N, Addington-Hall J. End-of-life care and preferences for place of death among the oldest old: results of a population-based survey using VOICES-Short Form. J Palliat Med 2014;17:176-82.

12 Koffman J, Ho YK, Davies J, et al. Does ethnicity affect where people with cancer die? A population-based 10 year study. PLOS ONE 2014;9:e95052.
13 Powell AE, Davies HT, Thomson RG. Using routine comparative data to assess the quality of health care: understanding and avoiding common pitfalls. Qual Saf Health Care 2003; 12:122-8.

14 Velentgas P, Dreyer NA, Nourjah P, et al., eds. Developing a protocol for observational comparative effectiveness research: a user's guide. Rockville, MD: Agency for Healthcare Research and Quality (US), 2013.

15 Langan SM, Benchimol EI, Guttmann A, et al. Setting the RECORD straight: developing a guideline for the Reporting of studies Conducted using Observational Routinely collected Data. Clin Epidemiol 2013;5:29-31.

16 Stewart R. The big case register. Acta Psychiatr Scand 2014;130:83-6.

17 Gaye A, Marcon Y, Isaeva J, et al. DataSHIELD: taking the analysis to the data, not the data to the analysis. Int $J$ Epidemiol 2014;43:1929-44.

18 Ekstrom MP, Bornefalk-Hermansson A, Abernethy AP, et al. Safety of benzodiazepines and opioids in very severe respiratory disease: national prospective study. BMJ 2014;348:g445.

19 England NHS. Developing a new approach to palliaitve care funding. NHS England, 2015.

20 Currow D, Allingham S, Yates P, et al. Improving national hospice/palliative care service symptom outcomes systematically through point-of-care data collection, structured feedback and benchmarking. Support Care Cancer 2015;23: 307-15.

21 Casarett DJ, Harrold J, Oldanie B, et al. Advancing the science of hospice care: coalition of hospices organized to investigate comparative effectiveness. Curr Opin Support Palliat Care 2012;6:459-64. 